\title{
Spectroscopy of the post-AGB star HD 101584 (IRAS $11385-5517)^{\star}$
}

\author{
T. Sivarani ${ }^{1}$, M. Parthasarathy ${ }^{1}$, P. García-Lario ${ }^{2}$, A. Manchado ${ }^{3}$, and S.R. Pottasch ${ }^{4}$ \\ 1 Indian Institute of Astrophysics, Bangalore 560 034, India \\ 2 ISO Data Centre, Astrophysics Division, Space Science Department of ESA, Villafranca del Castillo, Apartado de Correos \\ 50727, E-28080 Madrid, Spain \\ 3 Instituto de Astrofisica de Canarias, E-38200 La Laguna, Tenerife, Spain \\ ${ }^{4}$ Kapteyn Astronomical Institute, Postbus 800, NL-9700 AV Groningen, The Netherlands
}

Received October 22, 1998; accepted May 12, 1999

\begin{abstract}
From an analysis of the spectrum (4000 $\AA$ to $8800 \AA$ ) of HD 101584 it is found that most of the neutral and single ionized metallic lines are in emission. The forbidden emission lines of [OI] $6300 \AA$ and $6363 \AA$ and [CI] $8727 \AA$ are detected, which indicate the presence of a very low excitation nebula. The $\mathrm{H} \alpha$, FeII $6383 \AA$, NaI D $1, \mathrm{D}_{2}$ lines and the CaII IR triplet lines show P-Cygni profiles indicating a mass outflow. The $\mathrm{H} \alpha$ line shows many velocity components in the profile. The FeII $6383 \AA$ also has almost the same line profile as the $\mathrm{H} \alpha$ line indicating that they are formed in the same region. From the spectrum synthesis analysis we find the atmospheric parameters to be $T_{\text {eff }}=8500 \mathrm{~K}, \log g=1.5, V_{\text {turb }}=13 \mathrm{~km} \mathrm{~s}^{-1}$ and $[\mathrm{Fe} / \mathrm{H}]=0.0$. From an analysis of the absorption lines the photospheric abundances of some of the elements are derived. Carbon and nitrogen are found to be overabundant. From the analysis of Fe emission lines we derived $T_{\text {exi }}=6100 \mathrm{~K} \pm 200$ for the emission line region.
\end{abstract}

Key words: stars: abundances-stars: evolution-stars: post-AGB-stars: circumstellar matter-stars: individual: HD 101584

\section{Introduction}

Humphreys \& Ney (1974) found near-infrared excess in HD 101584 and suggested that it is a massive F-supergiant with an M-type binary companion star (Humphreys 1976).

Send offprint requests to: T. Sivarani

* Based on observations obtained at the European Southern Observatory (ESO), Chile and the Vainu Bappu Observatory, Kavalur, India.

Correspondence to: sivarani@iiap.ernet.in
However, HD 101584 ( $V=7.01$, F0 Iape (Hoffleit et al. 1983) was found to be an IRAS source (IRAS 11385-5517) (Parthasarathy \& Pottasch 1986). On the basis of its farinfrared colors, flux distribution and detached cold circumstellar dust shell, Parthasarathy \& Pottasch (1986) suggested that it is a low mass star in the post-Asymptotic Giant Branch (post-AGB) stage of evolution.

CO molecular emission lines at millimeter wavelengths were detected by Trams et al. (1990). The complex structure of the CO emission shows large Doppler velocities of $130 \mathrm{~km} \mathrm{~s}^{-1}$ with respect to the central velocity of the feature indicating a very high outflow velocity. Te Lintel Hekkert et al. (1992) reported the discovery of $\mathrm{OH} 1667$ $\mathrm{MHz}$ maser emission from the circumstellar envelope of HD 101584. The $\mathrm{OH}$ spectrum has a velocity range of $84 \mathrm{~km} \mathrm{~s}^{-1}$ and shows two unusually broad emission features. Te Lintel Hekkert et al. (1992) found from the images obtained from the Australian Telescope, that the $\mathrm{OH}$ masers are located along the bipolar outflow. The postAGB nature of HD 101584 is also suggested by the space velocity of the star derived from the central velocity of the $\mathrm{CO}$ and $\mathrm{OH}$ line emission. This velocity of $V_{\mathrm{rad}}=50.3$ $\pm 2.0 \mathrm{~km} \mathrm{~s}^{-1}$ does not agree with the galactic rotation curve assuming it to be a luminous massive population I F supergiant.

Bakker et al. (1996a) studied the low and high resolution ultraviolet spectra and the high resolution optical spectra of HD 101584. Based on the strength of HeI (see also Morrison \& Zimba 1989) N II, C II lines and Geneva photometry, Bakker et al. (1996a) suggest that HD 101584 is a B9 II star of $T_{\text {eff }}=12000 \mathrm{~K} \pm 1000 \mathrm{~K}$ and $\log g=3.0$. Bakker et al. (1996b) also found small amplitude light and velocity variations and suggested that HD 101584 is a binary with an orbital period of 218 days. 

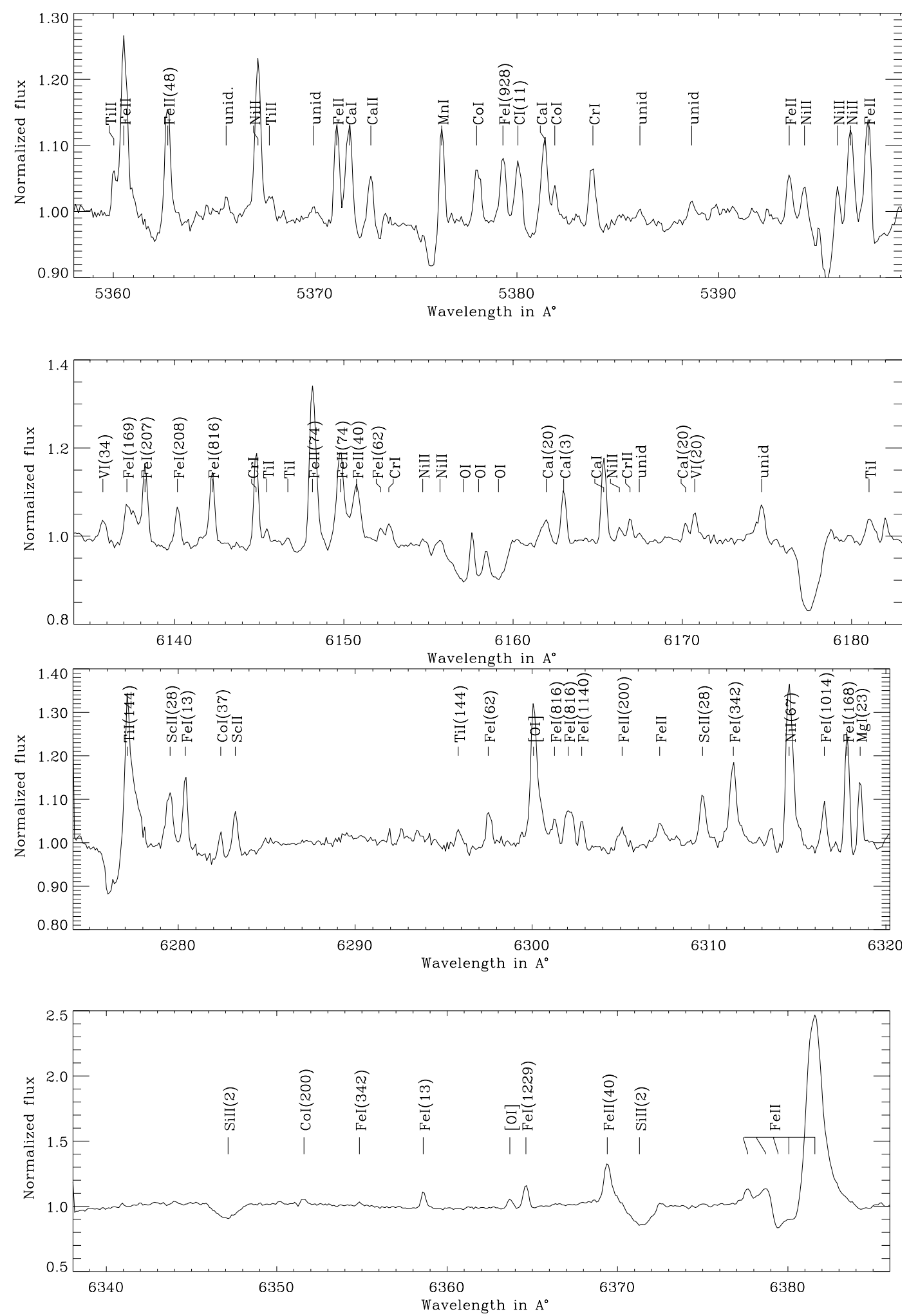

Fig. 1. High resolution spectra of HD 101584 obtained with the ESO CAT-CES 

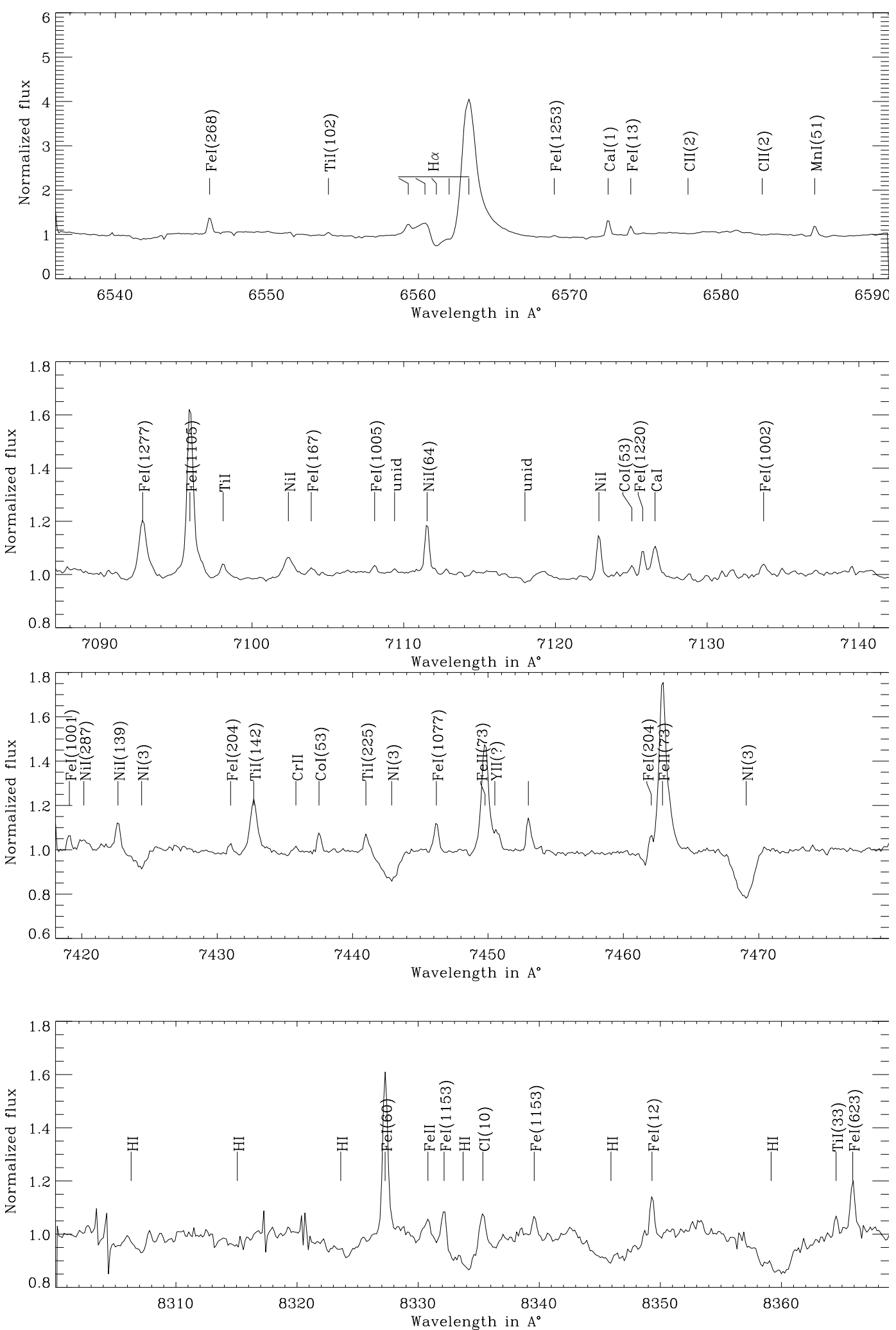

Fig. 1. continued 


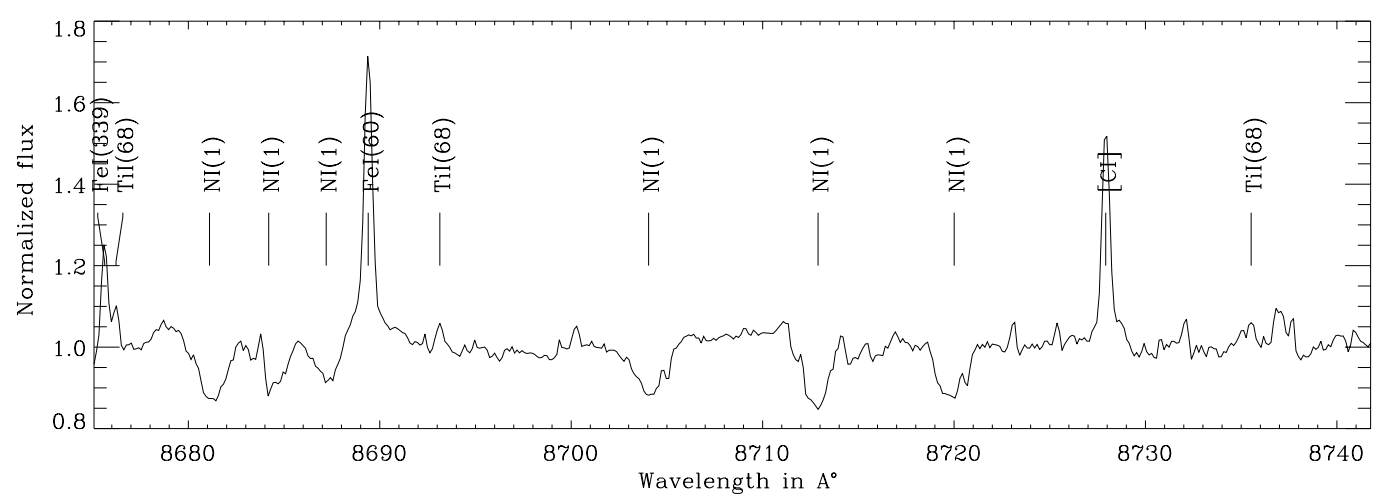

Fig. 1. continued
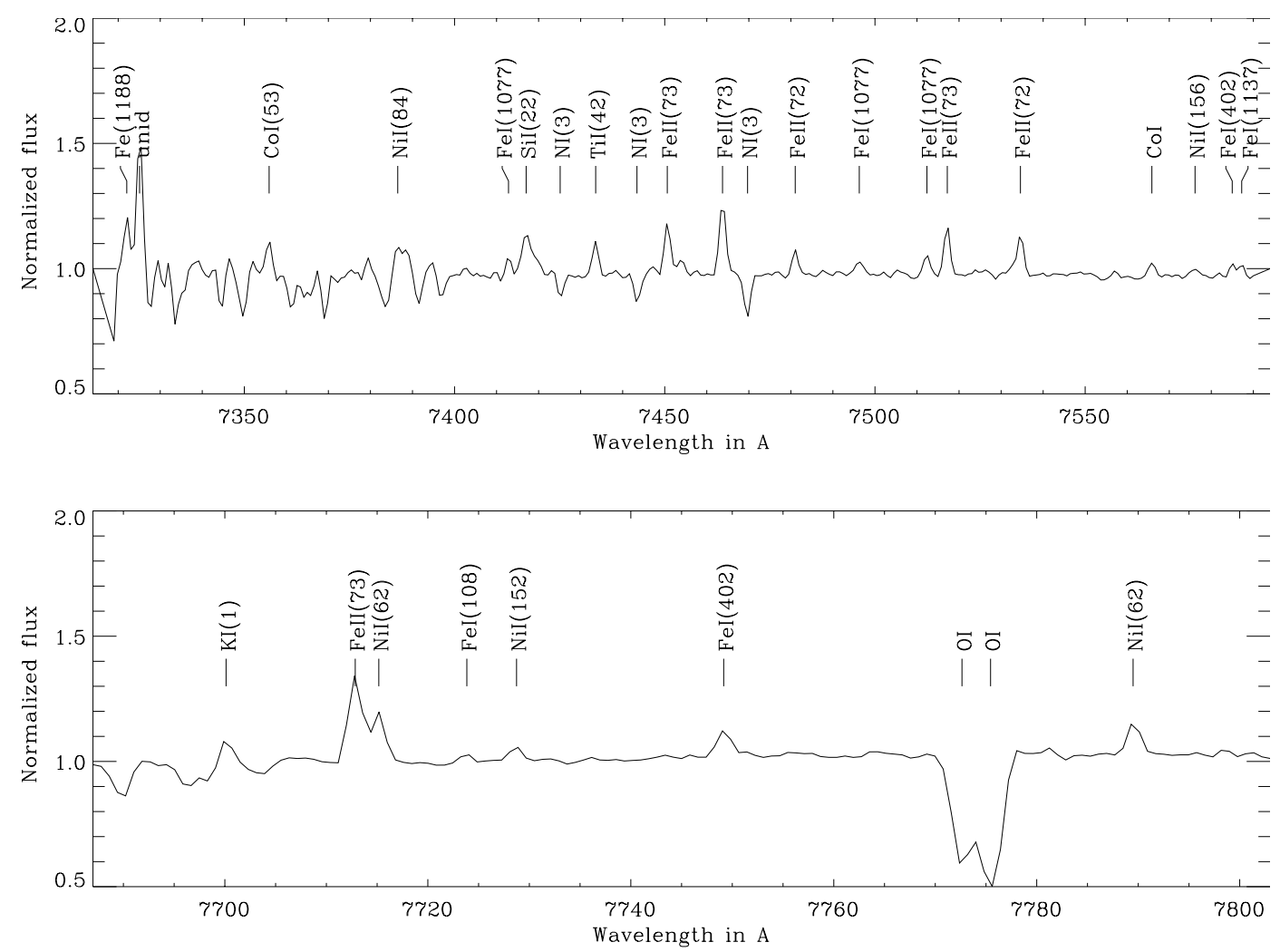

Fig. 2. The spectrum in the upper panel shows several nitrogen lines and emission lines of Fe. The lower panel shows the KI $7699 \AA$ in emission and the strong absorption due to OI triplet at $7777 \AA$

The optical spectrum of HD 101584 is very complex and shows many lines in emission. In this paper we report an analysis of the high resolution optical spectrum of HD 101584.

\section{Observations and analysis}

High resolution and high signal to noise ratio spectra of HD 101584 were obtained with the European Southern Observatory (ESO) Coude Auxiliary Telescope (CAT) equipped with the Coude Echelle Spectrograph (CES) and a CCD as detector. The spectra cover the wavelength regions $5360-5400 \AA, 6135-6185 \AA, 6280-6320 \AA$, $6340-6385 \AA, 6540-6590 \AA, 7090-7140 \AA, 7420-7480 \AA$, $8305-8365 \AA$ and $8680-8740 \AA$. The spectral resolution ranged from $0.165 \AA$ at $6150 \AA$ to $0.210 \AA$ at $8700 \AA$. We have also obtained $2.5 \AA$ resolution spectra of HD 101584 from $3900 \AA$ to $8600 \AA$ with the $1 \mathrm{~m}$ telescope and UAGS spectrograph and a CCD as detector at the Vainu Bappu Observatory (VBO), Kavalur, India. In addition we obtained CCD spectra with the same telescope and Coude Echelle spectrograph, covering the wavelength region $4600 \AA$ to $6600 \AA$ with a resolution of $0.4 \AA$. All spectra mentioned above were used in this analysis. 
T. Sivarani et al.: Spectroscopy of the post-AGB star HD 101584 (IRAS $11385-5517$ )

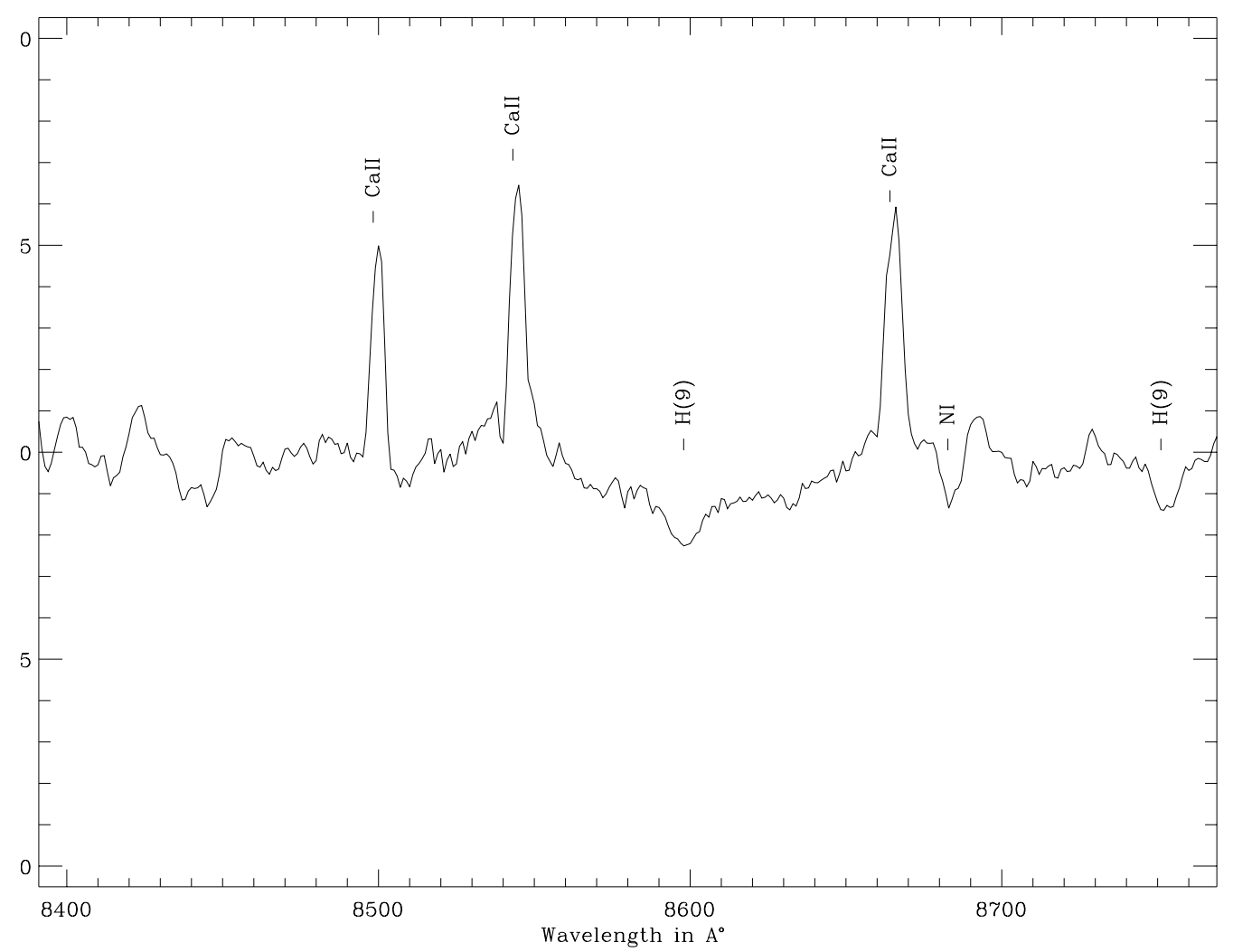

Fig. 3. CaII IR triplet lines showing P-Cygni emission.This spectrum is of $2.5 \AA$ resolution, obtained from VBO, Kavalur

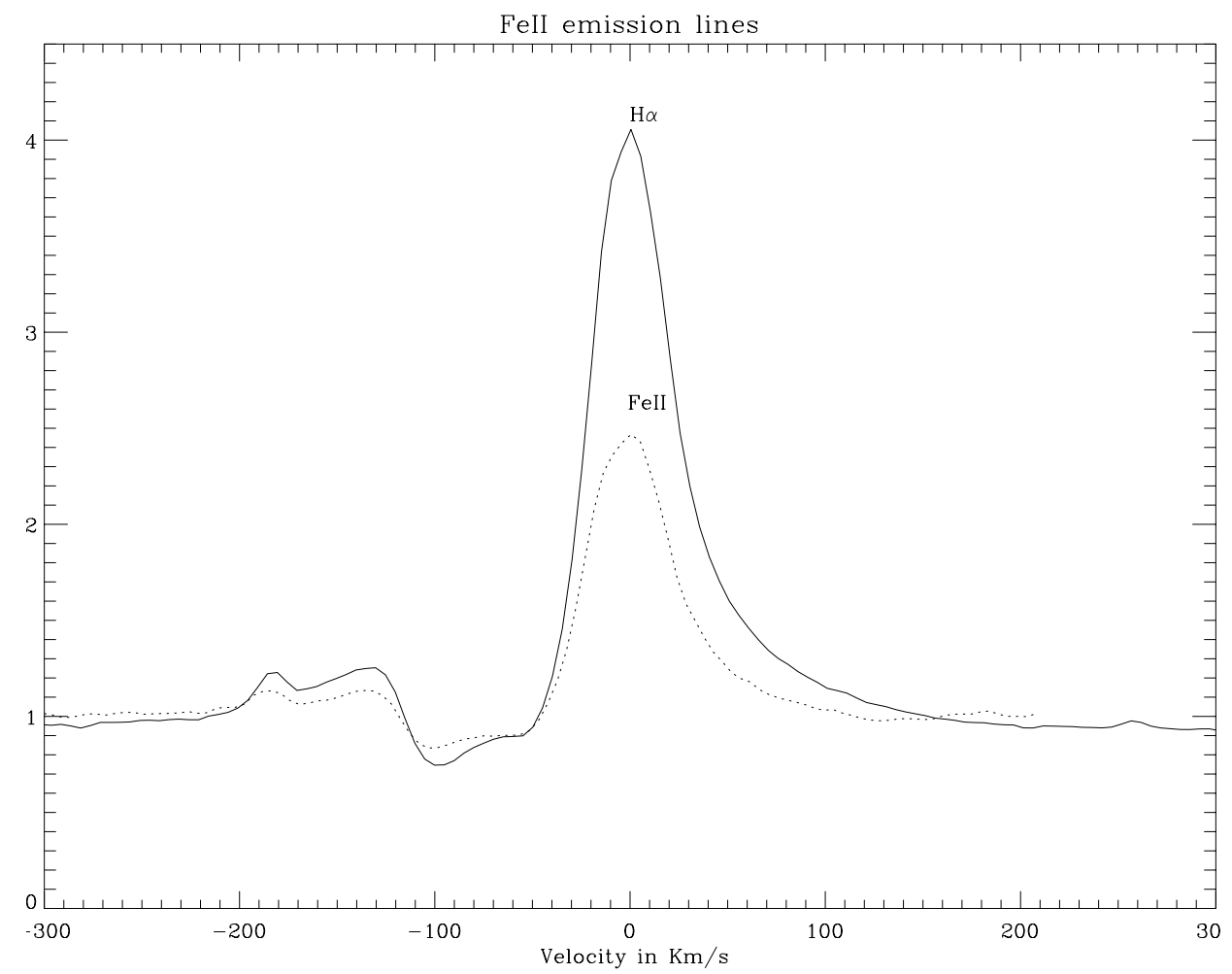

Fig. 4. P-Cygni profile of $\mathrm{H} \alpha$ and $\mathrm{FeII}(6383 \AA)$ lines showing similar velocity structures 


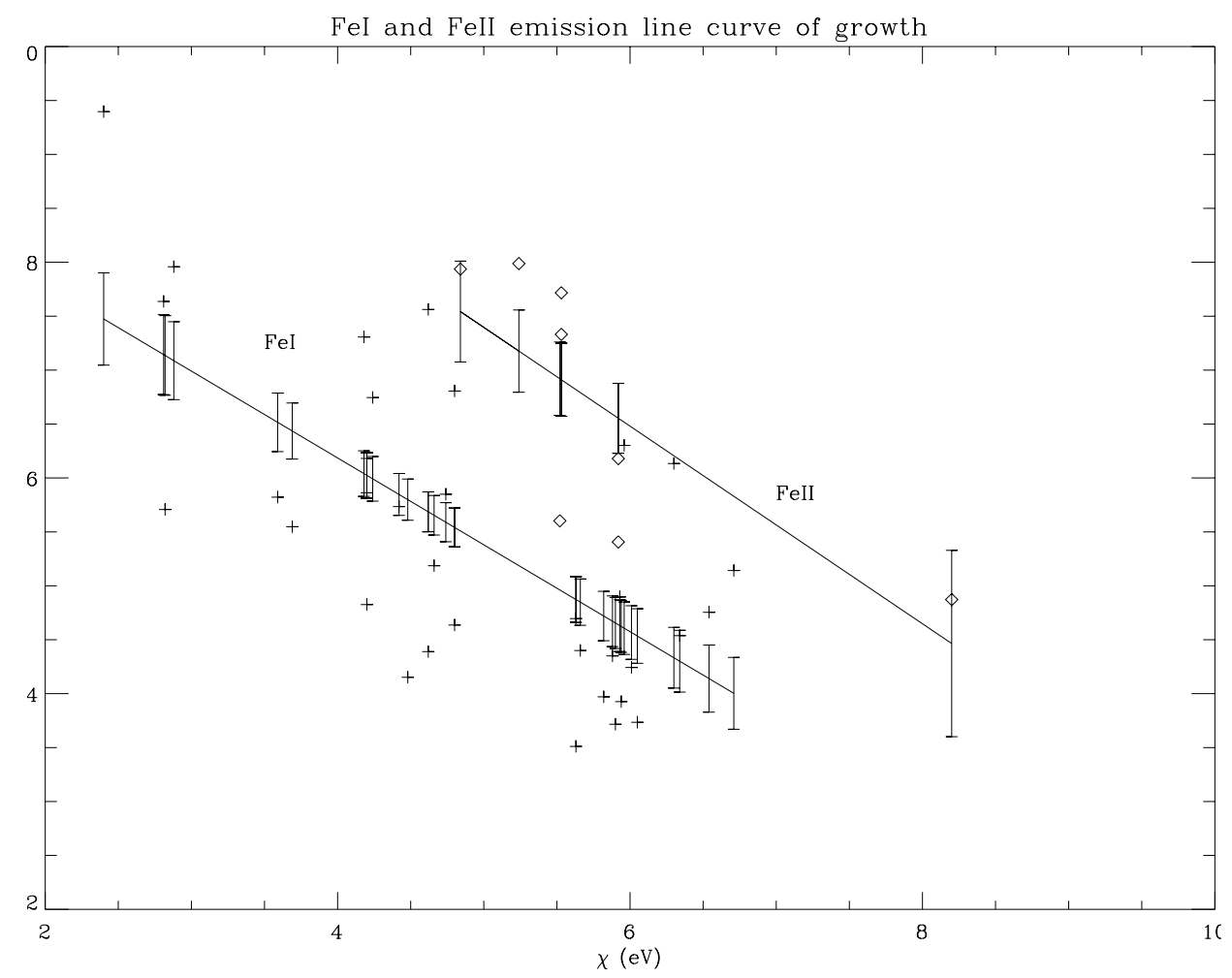

Fig. 5. Curve of growth analysis of Fe emission lines. + represents the FeI lines and $\diamond$ represents FeII lines. The slope gives $T_{\text {exi }}=6300 \pm 1000 \mathrm{~K}$ for FeI lines and $T_{\text {exi }}=5550 \pm 1700 \mathrm{~K}$ for the FeII lines. The large dispersion is because the lines are optically thick. The errors bar show the error in the least square fit

All the spectra were analyzed using IRAF software. The equivalent widths of lines were found by fitting a Gaussian. For blended lines de-blending was done by fitting multiple Gaussians. We carried out spectrum synthesis calculations using KURUCZ stellar models (1994). SYNSPEC code (Hubeny et al. 1985) was used for calculating the theoretical line profiles. The $g f$ values were taken from Wiese et al. (1966), Wiese \& Martin (1980), Hibbert et al. (1991), Parthasarathy et al. (1992) and Reddy et al. (1997 and references therein). For the analysis of forbidden lines we have used the IRAF software package NEBULAR under STSDAS.

\section{Description of the spectrum}

The remarkable characteristic of the optical spectrum of HD 101584 is the fact that different spectral regions resemble different spectral types. The spectrum in the UV region is similar to that of $\alpha$ Lep which is an F-supergiant (Bakker 1994). The optical spectrum in the range $3600 \AA$ - $5400 \AA$ is dominated by absorption lines. Most of them are due to neutral and single ionized lines of $\mathrm{Ti}, \mathrm{Cr}$ and Fe. The CaII $\mathrm{H}$ and $\mathrm{K}$ absorption lines are strong. The strength of the absorption lines are similar to that observed in an A2 supergiant. In the yellow and red spectral regions, most of the lines are in emission (Fig. 1).
The emission lines show complex line profiles. The absorption lines of NI, OI, CII and SiII are broad. The Paschen lines are in absorption. Some of these absorption lines are blended with emission lines and many have asymmetric profiles. The OI lines at $6156 \AA$ are blended with emission lines of FeI. The NI lines are strong and show asymmetric line profiles. The blue wing is shallow compared to the red wing. The CII lines at $6578 \AA$ and $6582 \AA$ are weak. The Na D lines, KI $7700 \AA$ (Fig. 2), the CaII IR triplet lines (Fig. 3), [OI], [CI] and MgI 6318.7 $\AA$ lines are found in emission. The OI triplet lines (Fig. 2) are very strong indicating an extended atmosphere and NLTE effects.

\subsection{P-Cygni profiles}

The $\mathrm{H} \alpha$ line has a very strong $\mathrm{P}-\mathrm{Cygni}$ profile indicating an outflow. The profile looks very complex. It shows at least 6 velocity components. The FeII line at $6383 \AA$ is in emission and the profile is very similar to that of $\mathrm{H} \alpha$ (Fig. 4). Similar behaviour of the $6383 \AA$ FeII line and $\mathrm{H} \alpha$ line is also noticed in the post-AGB F supergiant IRAS $10215-5916$ (García-Lario et al. 1994). The $\mathrm{H} \alpha$ and the FeII $6383 \AA$ line show an outflow velocity of $100 \pm 10 \mathrm{~km} \mathrm{~s}^{-1}$. The $\mathrm{H} \beta$ line also shows a P-Cygni profile. It has a broad emission wing at the red end. This indicates that the line forming region is extended. The $\mathrm{H} \beta$, 


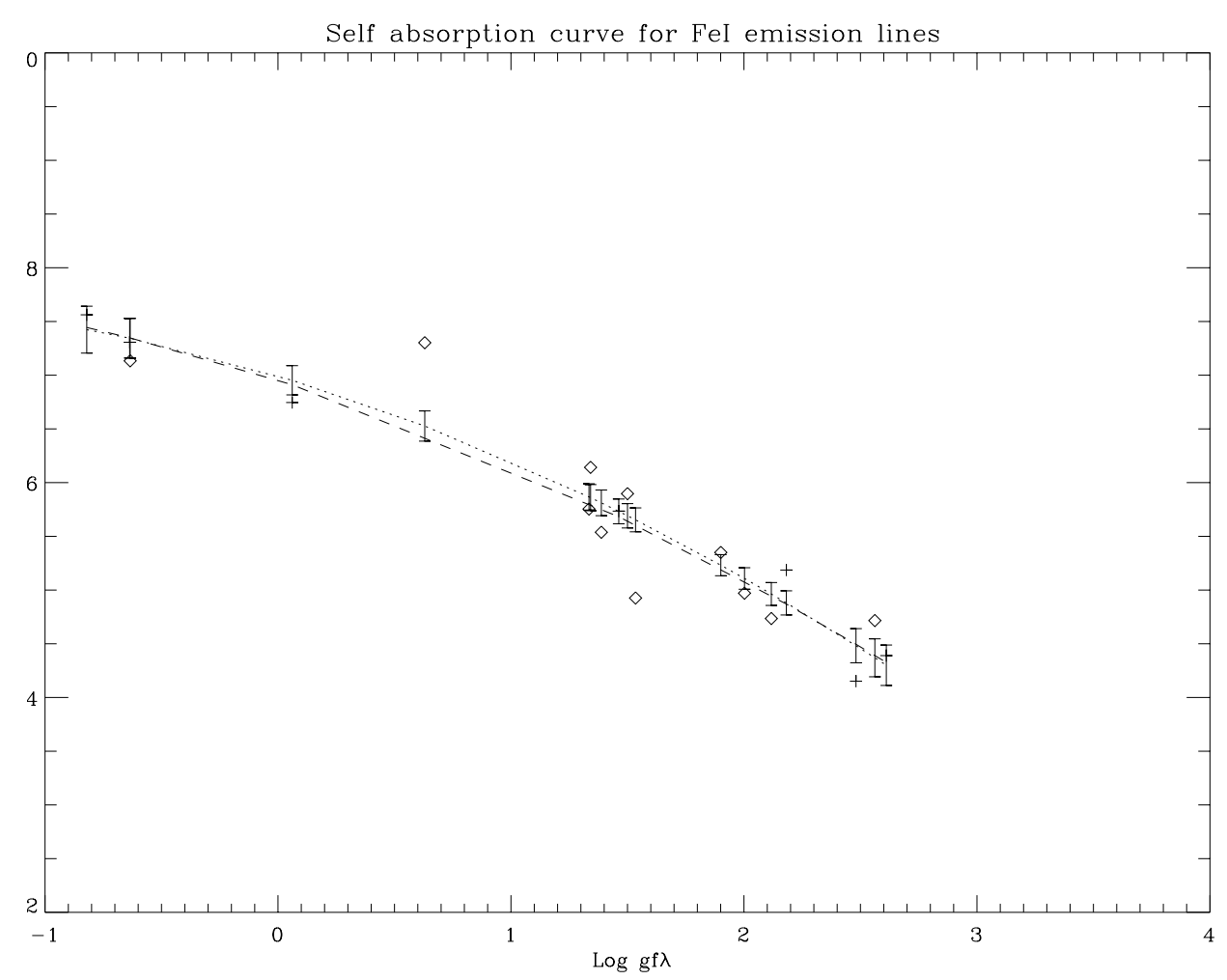

Fig. 6. a) The plot shows the shape of the SAC. The + sign indicates multiplets 167, 168, 169, 204, 207, 208 of Fe I having similar excitation potential. $\diamond$ indicates multiplets $1002,1005,1014,1077,1105,1140,1153,1220,1229,1277$ of FeI. The fit was obtained after shifting higher multiplets 1002, 1005, 1014, 1077, 1105, 1140, 1153, 1220, 1229, 1277 w.r. to the lower multiplets 167, 168, 169, 204, 207, 208

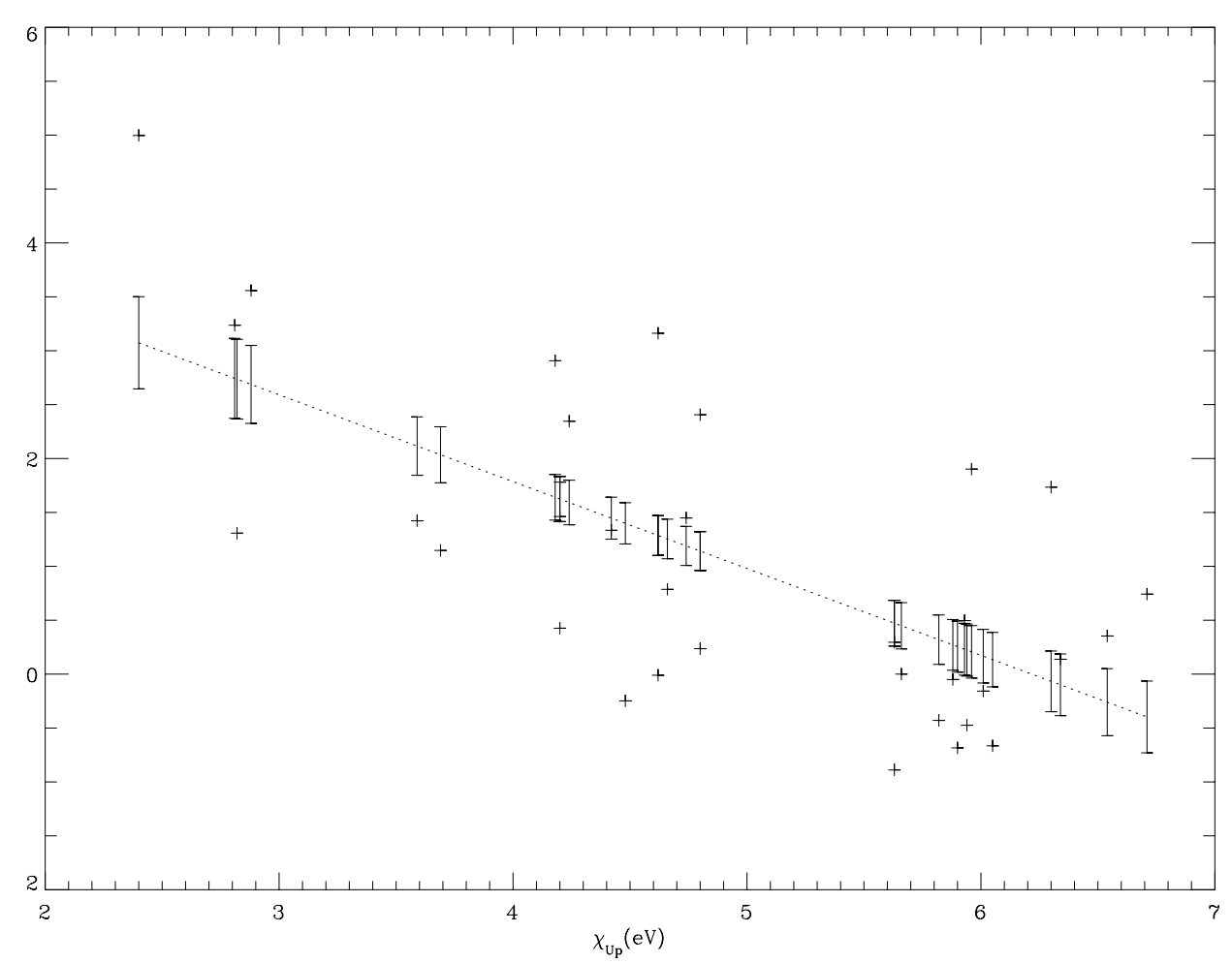

Fig. 6. b) The fit shows the distribution of upper level population of different multiplets of FeI with respect to the multiplet 207, versus the upper excitation potential 


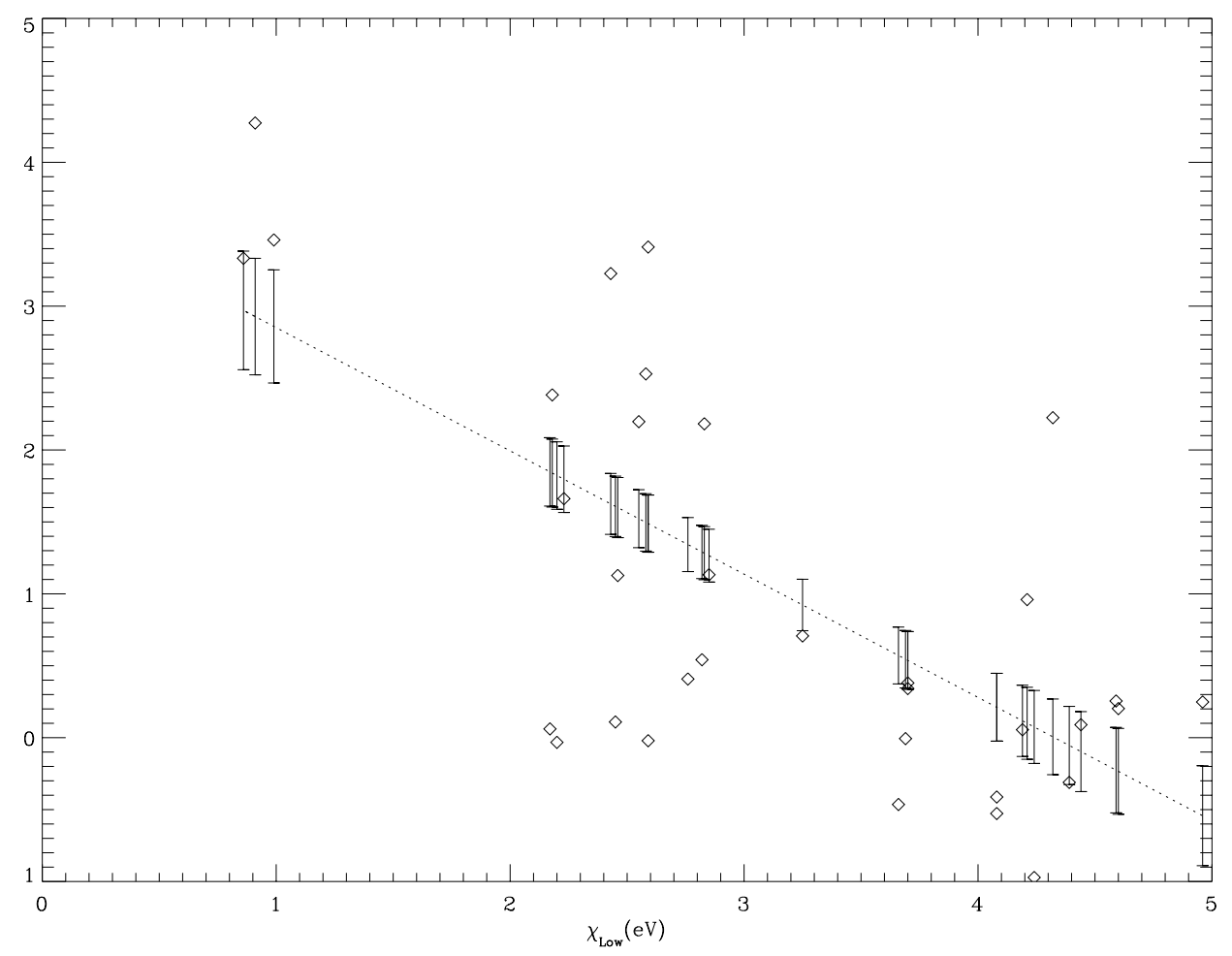

Fig. 6. c) The fit shows the distribution of lower level population of different multiplets of FeI with respect to the multiplet 207, versus the lower excitation potential

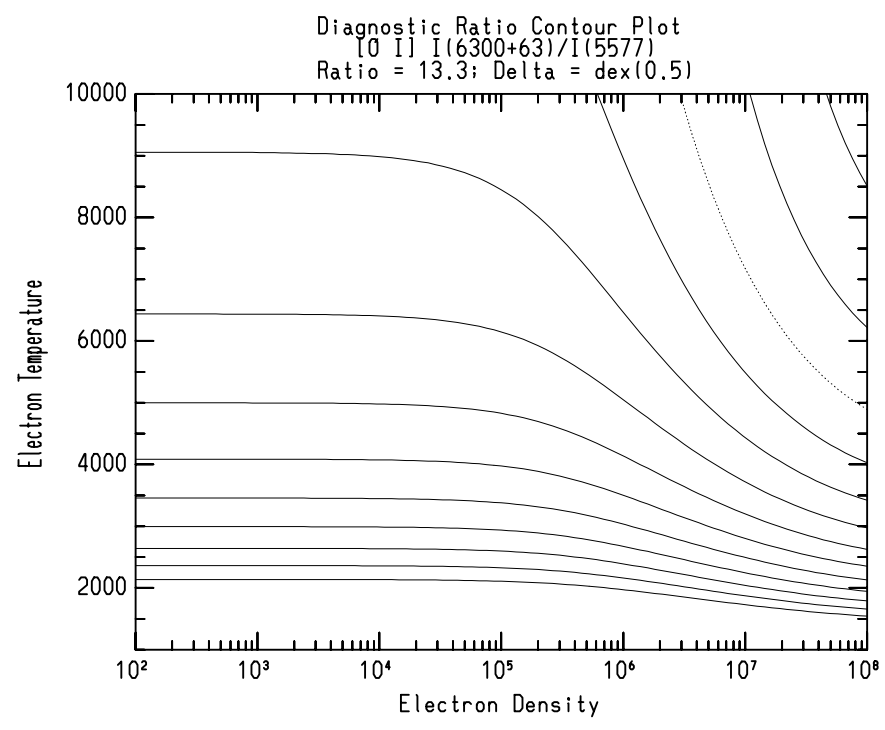

Fig. 7. Plot of electron density $N_{\mathrm{e}}$ and electron temperature $T_{\mathrm{e}}$. The dotted line is the contour for the observed ratio (13.3) of [O]I lines $5577 \AA, 6300 \AA$ and $6363 \AA$. Each contour in the plot is for a change in the flux ratio of 0.5

NaI D1, D2 and the CaII IR triplet lines (Fig. 3) show an outflow velocity of $75 \pm 20 \mathrm{~km} \mathrm{~s}^{-1}$. The velocity structure seen in these P-Cygni profiles could be due to emission from different shells formed during the episodic mass-loss events.

\subsection{FeI and FeII emission lines}

The presence of numerous emission lines of FeI and FeII makes it possible to derive the physical conditions of the line forming region. From the curve of growth analysis of the FeI and FeII emission lines (Viotti 1969), we have derived $T_{\text {exi }}=6300 \pm 1000 \mathrm{~K}$ and $5550 \pm 1700 \mathrm{~K}$ respectively (Fig. 5). The scatter found could be due to the fact that the lines are not optically thin. On the other hand, there are only few emission lines of FeII present in the spectra and thus the estimate from FeII might not be accurate. In order to determine whether the large scatter observed in Fig. 4 is reflecting optical thickness effects we have done self-absorption curve (SAC) analysis (Friedjung \& Muratorio 1987) for the FeI emission lines.

$\mathrm{SAC}$ is a kind of curve of growth applied to emission lines, but it has certain advantages as compared to the classical emission line curve of growth analysis. This method of analysis is valid also for optically thick lines. It deals with each transition separately, so that it is possible to get the population of different levels without assuming a Boltzmann distribution. In this curve, a function of the line flux emitted in the different transition of a given multiplet is taken in such a way that it is constant for a optically thin uniform medium. As the optical thickness increases the curve will move towards a straight line inclined at $-45^{\circ}$. The shape of the SAC in Fig. 6a shows the lines are optically thick. The shape of the SAC is obtained 

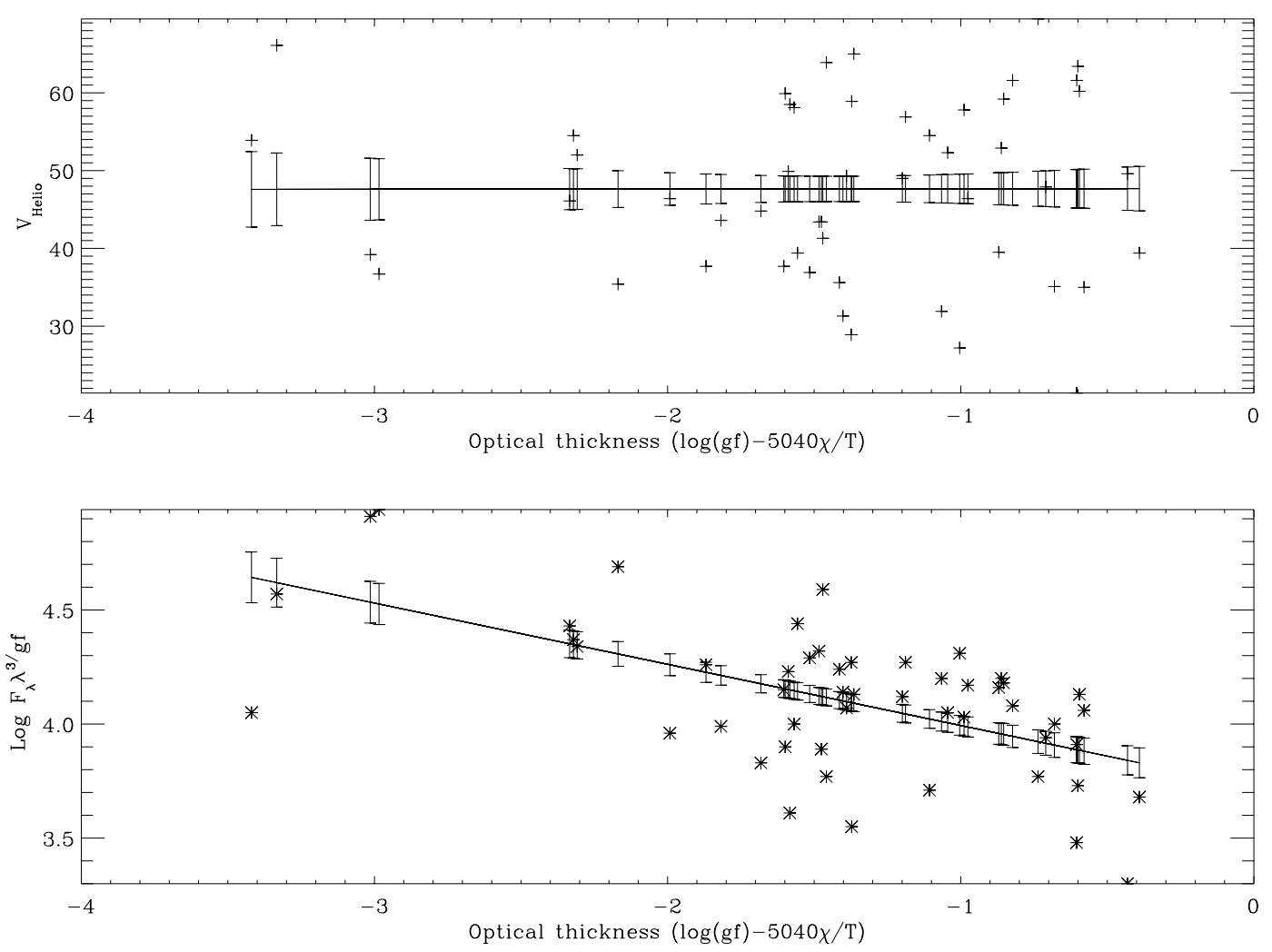

Fig. 8. The plot in the upper panel does not show any correlation between the optical depth and the helio centric radial velocity for the FeI absorption lines of HD 101584 in the wavelength region $3600 \AA-4500 \AA$. The plot in the lower panel shows the normalized strength of FeI absorption lines in the wavelength region $3600 \AA$ to $4500 \AA$ versus the optical depth. It shows clearly that the lines are forming at different optical depths. The equivalent widths are taken from the paper by Rosenzweig et al. (1997)

by shifting all the multiplets with respect to a reference multiplet. Here we have taken multiplet 207 as reference. The $X$ and $Y$ shifts of each multiplet gives the relative population of the lower and upper level with respect to the reference multiplet. Figures $6 \mathrm{~b}, \mathrm{c}$ shows the $Y$ and $X$ shifts versus the upper and lower excitation potential from which we derive the $T_{\text {exi }}=6100 \pm 200 \mathrm{~K}$.

\subsection{Forbidden lines}

The forbidden emission lines at $5577 \AA$, $6300 \AA$ and $6363 \AA$ of neutral oxygen are present in the spectra. The forbidden line of neutral carbon at $8727 \AA$ is also seen. The $6300 \AA$ line is blended with ScII line and the $5577 \AA$ line is very weak. We have calculated the $I(6300)+I(6363) / I(5577)$ to be 13.3. From the flux ratio we can calculate $T_{\mathrm{e}}$ (Osterbrock 1989). This flux ratio is not very accurate because of very the weak $5577 \AA$ line and poor signal to noise spectrum. For the flux ratio of 13.3 we derived a function depending on the electron density $N_{\mathrm{e}}$ and temperature $T_{\mathrm{e}}$. Figure 7 shows the $N_{\mathrm{e}}$ and $T_{\mathrm{e}}$ contours for different values of flux ratio around 13.3. Since we do not see any other forbidden lines which are sensitive to the electron density, we could not fix both $N_{\mathrm{e}}$ and $T_{\mathrm{e}}$ uniquely. But assuming a temperature derived from the Fe emission lines, an electron density of $110^{7}$ is obtained. For this value of electron density and temperature the $\mathrm{C} / \mathrm{O}=0.5 \pm 0.2$ has been obtained.

\section{Radial velocities}

There are very few absorption lines and most of these are affected by emission and or a shell component, therefore we derived the average radial velocity from the well defined emission lines. The average radial velocity from the emission lines is found to be $50 \pm 2 \mathrm{~km} \mathrm{~s}^{-1}$. Morrison \& Zimba (1989) using 14 best absorption lines found the radial velocity to be $69 \pm 1 \mathrm{~km} \mathrm{~s}^{-1}$. From the equivalent widths of FeI absorption lines given by Rosenzweig et al. (1997) we find no correlation between log $g f-\chi \Theta$ and heliocentric radial velocity (Fig. 8). However, Bakker et al. (1996a) found a correlations between $\log g f-\chi \Theta$ and heliocentric radial velocities of HD 101584 in the UV. The discripancy could be due to the poor resolution of Rosenzweig et al. (1997) data compared to that of Bakker et al. (1996a). The large scatter seen in the radial velocities could be due to pulsation. Similar velocity variations 
Table 1. List of emission lines detected in the high resolution spectrum of HD 101584

\begin{tabular}{|c|c|c|c|c|c|c|c|}
\hline $\begin{array}{l}\lambda \text { obs } \\
(\AA)\end{array}$ & $\begin{array}{l}\lambda \text { lab } \\
(\AA)\end{array}$ & Ident. & $\begin{array}{c}\chi \\
(\mathrm{eV})\end{array}$ & $\log g f$ & $\begin{array}{l}F_{\lambda}{ }^{*} \\
(\mathrm{~m} \AA)\end{array}$ & $\begin{array}{l}\Delta \lambda \\
(\AA)\end{array}$ & $\begin{array}{r}V_{\text {Helio }} \\
\mathrm{km} \mathrm{s}^{-1}\end{array}$ \\
\hline 5360.022 & 5360.115 & TiII & $2.60-4.92$ & -4.040 & & -0.093 & 10.60 \\
\hline 5360.517 & 5360.755 & FeII & $10.52-12.83$ & -3.082 & 118.4 & -0.237 & 2.51 \\
\hline 5362.698 & 5362.864 & $\mathrm{FeII}(48)$ & $3.20-5.52$ & -2.739 & 47.36 & -0.165 & 6.56 \\
\hline 5365.597 & & unid & & & & & \\
\hline 5367.167 & 5367.205 & NiII & $14.61-16.93$ & -2.571 & 79.71 & -0.038 & 13.69 \\
\hline 5367.731 & 5367.912 & TiII & $1.57-3.88$ & -4.480 & & -0.181 & 5.69 \\
\hline 5369.928 & 5369.928 & unid & & & & & \\
\hline 5371.07 & 5371.275 & FeII & $10.56-12.87$ & -0.705 & 45.52 & -0.20 & 4.36 \\
\hline 5371.704 & 5371.934 & $\mathrm{CaI}$ & $5.4-7.71$ & -3.163 & & -0.220 & 2.97 \\
\hline 5372.76 & 5372.807 & $\mathrm{CaII}$ & $9.03-11.35$ & -1.337 & 21.64 & -0.04 & 13.17 \\
\hline 5376.27 & 5376.477 & MnI & $2.93-5.23$ & -4.091 & 45.13 & -0.20 & 4.26 \\
\hline 5378. & 5378.247 & $\mathrm{CoI}$ & $4.03-6.34$ & -0.425 & 32.41 & -0.24 & 2.03 \\
\hline 5379.305 & 5379.581 & $\mathrm{FeI}(928)$ & $3.70-6.01$ & -1.521 & 33.83 & -0.275 & 0.43 \\
\hline 5380.048 & 5380.322 & $\mathrm{CI}(11)$ & $7.70-10.00$ & -1.840 & 32.67 & -0.273 & 0.54 \\
\hline 5381.379 & 5381.599 & $\mathrm{CaI}$ & $5.46-7.77$ & -4.960 & 49.97 & -0.220 & 3.54 \\
\hline 5381.874 & 5382.046 & $\mathrm{CoI}$ & $4.51-6.82$ & -3.234 & 15.82 & -0.171 & 6.24 \\
\hline 5383.763 & 5383.927 & $\mathrm{CrI}$ & $4.54-6.85$ & -2.606 & 22.12 & -0.163 & 6.70 \\
\hline 5386.093 & 5386.093 & unid & & & & & \\
\hline 5388.659 & 5388.659 & unid & & & & & \\
\hline 5393.491 & 5393.762 & FeII & $11.19-13.49$ & -2.154 & 17.12 & -0.270 & 0.74 \\
\hline 5394.247 & 5394.496 & NiII & $12.64-14.94$ & -1.029 & 14.61 & -0.249 & 1.97 \\
\hline 5395.886 & 5396.051 & NiII & $14.63-16.93$ & -2.851 & 10.14 & -0.164 & 6.67 \\
\hline 5396.532 & 5396.701 & NiII & $14.63-16.93$ & -2.561 & 54.78 & -0.168 & 6.42 \\
\hline 5397.4 & 5397.640 & FeII & $10.73-13.03$ & -2.883 & 51.29 & -0.240 & 2.46 \\
\hline 6133.723 & 7132.985 & $\mathrm{FeI}(1002)$ & $4.08-5.82$ & -0.851 & 36.31 & 0.738 & 46.96 \\
\hline 6135.76 & 6135.370 & $\mathrm{VI}(34)$ & $1.05-3.08$ & -0.750 & 23.13 & 0.389 & 35.22 \\
\hline 6137.179 & 6136.624 & $\mathrm{FeI}(169)$ & $2.45-4.48$ & -1.307 & 30.29 & 0.555 & 43.31 \\
\hline 6138.25 & 6137.702 & $\mathrm{FeI}(207)$ & $2.59-4.62$ & -1.177 & 70.63 & 0.547 & 42.95 \\
\hline 6140.167 & 6139.65 & $\mathrm{FeI}(208)$ & $2.59-4.62$ & -4.609 & 38.79 & 0.517 & 41.43 \\
\hline 6142.214 & 6141.727 & $\mathrm{FeI}(816)$ & $3.70-5.63$ & -1.540 & 61.82 & 0.486 & 39.95 \\
\hline 6154.659 & 6154.094 & NiII & $14.88-16.91$ & -2.268 & 5.54 & 0.564 & 43.71 \\
\hline 6144.813 & 6144.294 & $\mathrm{CrI}$ & $4.10-6.13$ & -3.624 & 80.21 & 0.519 & 41.51 \\
\hline 6145.448 & 6144.936 & TiI & $1.89-3.91$ & -2.520 & 16.08 & 0.512 & 41.17 \\
\hline 6146.688 & 6146.27 & TiI(153) & $3.18-5.20$ & -2.541 & 15.79 & 0.417 & 36.57 \\
\hline 6148.146 & 6147.742 & FeII(74) & $3.89-5.92$ & -2.721 & 20.85 & 0.403 & 35.87 \\
\hline 6149.804 & 6149.249 & $\operatorname{FeII}(74)$ & $3.89-5.92$ & -2.724 & 122.7 & 0.555 & 43.25 \\
\hline 6150.747 & 6150.10 & FeII(40) & $3.22-5.24$ & -4.754 & 73.71 & 0.646 & 47.73 \\
\hline 6152.178 & 6151.624 & $\mathrm{FeI}(62)$ & $2.18-4.20$ & -3.582 & 17.06 & 0.554 & 43.20 \\
\hline 6152.655 & 6152.098 & $\mathrm{CrI}$ & $4.10-6.12$ & -1.986 & 33.16 & 0.556 & 43.31 \\
\hline 6155.679 & 6155.102 & NiII & $13.14-15.16$ & -0.682 & 7.19 & 0.577 & 44.30 \\
\hline 6161.957 & 6161.295 & $\mathrm{CaI}(20)$ & $2.53-4.54$ & -1.293 & 27.97 & 0.662 & 48.41 \\
\hline 6162.99 & 6162.180 & $\mathrm{CaI}(3)$ & $1.90-3.92$ & -0.167 & 49.42 & 0.810 & 55.61 \\
\hline 6165.354 & 6164.716 & $\mathrm{CaI}$ & $6.05-8.07$ & -2.261 & 73.94 & 0.638 & 47.22 \\
\hline 6166.282 & 6165.893 & NiII & $9.75-11.77$ & -2.297 & 9.67 & 0.389 & 35.10 \\
\hline 6166.907 & 6166.187 & CrII & $13.06-15.07$ & -3.490 & 18.24 & 0.720 & 51.21 \\
\hline 6167.456 & 6167.456 & unid & & & 10.3 & & \\
\hline 6170.189 & 6169.559 & $\mathrm{CaI}(20)$ & $2.53-4.54$ & -0.527 & 13.17 & 0.629 & 46.80 \\
\hline 6170.733 & 6170.340 & $\mathrm{VI}(20)$ & $4.80-6.81$ & -0.654 & 23.53 & 0.216 & 26.71 \\
\hline 6174.685 & 6174.685 & unid & & & 39.17 & & \\
\hline 6181.032 & 6180.625 & TiI & $3.18-5.19$ & -3.632 & 43.54 & 0.407 & 35.93 \\
\hline 6277.13 & 6277.470 & $\operatorname{TiI}(144)$ & $1.73-3.71$ & -3.794 & 112.2 & -0.205 & 6.20 \\
\hline 6279.541 & 6279.740 & $\operatorname{ScII}(28)$ & $1.5-3.48$ & -1.265 & 41.49 & -0.287 & 2.29 \\
\hline 6280.406 & 6280.622 & $\mathrm{FeI}(13)$ & $0.86-2.83$ & -3.720 & 44.44 & -0.216 & 5.67 \\
\hline 6282.404 & 6282.638 & $\mathrm{CoI}(37)$ & $1.74-3.72$ & -2.021 & 13.03 & -0.234 & 4.81 \\
\hline
\end{tabular}


Table 1. continued

\begin{tabular}{|c|c|c|c|c|c|c|c|}
\hline $\begin{array}{l}\lambda \text { obs } \\
(\AA)\end{array}$ & $\begin{array}{l}\lambda \text { lab } \\
(\AA)\end{array}$ & ID & $\begin{array}{l}\chi \\
(\mathrm{eV})\end{array}$ & $\log g f$ & $\begin{array}{l}F_{\lambda}{ }^{*} \\
(\mathrm{~m} \AA)\end{array}$ & $\begin{array}{l}\Delta \lambda \\
(\AA)\end{array}$ & $\begin{array}{r}V_{\text {Helio }} \\
\mathrm{km} \mathrm{s}^{-1}\end{array}$ \\
\hline 6283.225 & 6283.353 & ScII & $7.45-9.43$ & -0.441 & 40.34 & -0.127 & 9.90 \\
\hline 6295.826 & 6295.949 & $\operatorname{TiI}(144)$ & & & & & \\
\hline 6297.524 & 6297.799 & $\operatorname{FeI}(62)$ & $2.23-4.20$ & -2.871 & 39.23 & -0.274 & 2.91 \\
\hline 6300.083 & 6300.311 & {$[\mathrm{OI}]$} & & & 163 & -0.228 & 5.15 \\
\hline 6301.27 & 6301.508 & $\mathrm{FeI}(816)$ & $3.66-5.63$ & -0.745 & 23.32 & -0.237 & 4.68 \\
\hline 6302.042 & 6302.499 & $\mathrm{FeI}(816)$ & $3.69-5.66$ & -1.203 & 62.88 & -0.457 & -5.75 \\
\hline 6302.807 & 6303.461 & FeI(1140) & $4.32-6.30$ & -3.434 & 20.01 & -0.653 & -15.11 \\
\hline 6305.09 & 6305.314 & $\operatorname{FeII}(200)$ & $6.23-8.20$ & -2.039 & 27.17 & -0.224 & 5.34 \\
\hline 6307.214 & 6307.529 & FeII & $2.83-4.80$ & -5.685 & 57.4 & -0.314 & 1.02 \\
\hline 6309.636 & 6309.886 & $\operatorname{ScII}(28)$ & $1.5-3.47$ & -1.630 & 55.71 & -0.250 & 4.12 \\
\hline 6311.379 & 6311.504 & $\mathrm{FeI}(342)$ & $2.83-4.80$ & -3.392 & 103.2 & -0.125 & 10.06 \\
\hline 6313.52 & & & & & & & \\
\hline 6314.529 & 6314.668 & NiI(67) & $4.16-6.13$ & -0.921 & 163.5 & -0.139 & 9.39 \\
\hline 6316.52 & 6315.814 & $\mathrm{FeI}(1014)$ & $4.08-6.05$ & -0.683 & 44.72 & 0.706 & 49.54 \\
\hline 6317.802 & 6318.027 & $\mathrm{FeI}(168)$ & $2.46-4.42$ & -2.338 & 98.8 & -0.181 & 7.40 \\
\hline 6318.545 & 6318.717 & $\operatorname{MgI}(23)$ & $5.12-7.08$ & -1.730 & 60.1 & -0.171 & 7.84 \\
\hline 6351.598 & 6351.448 & $\mathrm{CoI}(200)$ & & & & & \\
\hline 6354.852 & 6355.027 & $\mathrm{FeI}(342)$ & $2.85-4.80$ & -2.346 & 7.6 & -0.174 & 7.62 \\
\hline 6358.596 & 6358.687 & $\operatorname{FeI}(13)$ & $0.86-2.81$ & -4.546 & 47.9 & -0.0908 & 11.59 \\
\hline 6363.68 & 6363.79 & {$[\mathrm{OI}]$} & & & 28.9 & -0.109 & 10.69 \\
\hline 6364.625 & 6364.706 & FeI(1229) & $4.59-6.54$ & -1.469 & 74.78 & -0.081 & 12.05 \\
\hline 6369.4 & 6369.464 & $\mathrm{FeII}(40)$ & $2.89-4.84$ & -4.253 & 186.8 & -0.063 & 12.86 \\
\hline 6380.058 & 6383.715 & FeII & $5.56-7.50$ & -2.271 & 1512 & -3.656 & -155.97 \\
\hline 6381.582 & 6381.416 & TiI(196) & & & & & \\
\hline 6539.783 & 6539.72 & $\mathrm{FeI}(405)$ & & & & & \\
\hline 6546.217 & 6546.245 & $\mathrm{FeI}(268)$ & $2.76-4.66$ & -1.634 & 127.2 & -0.0283 & 14.97 \\
\hline 6554.056 & 6554.226 & $\operatorname{TiI}(102)$ & $1.44-3.34$ & -1.201 & 32.48 & -0.169 & 8.49 \\
\hline 6561.179 & 6561.179 & $\mathrm{H} \alpha$ & & & 3681 & & \\
\hline 6563.326 & 6563.403 & $\mathrm{CoI}(8$ & & & & & \\
\hline 6568.959 & 6569.231 & $\mathrm{FeI}(125$ & & & & & \\
\hline 6572.512 & 6572.779 & $\mathrm{CaI}(1)$ & $0.00-1.89$ & -4.104 & 124.5 & -0.266 & 4.10 \\
\hline 6574.009 & 6574.238 & $\mathrm{FeI}(13)$ & $0.99-2.88$ & -4.688 & 65.55 & -0.229 & 5.82 \\
\hline 6574.831 & 6575.022 & $\mathrm{FeI}(207)$ & & & & & \\
\hline 6580.992 & 6581.220 & $\operatorname{FeI}(34)$ & & & & & \\
\hline 6586.144 & 6586.343 & $\operatorname{MnI}(51)$ & & & & & \\
\hline 6587.438 & 6587.75 & $\mathrm{CI}(22)$ & & & & & \\
\hline 7092.793 & 7091.942 & $\mathrm{FeI}(1277)$ & $4.96-6.71$ & -1.509 & 120.4 & 0.886 & 53.22 \\
\hline 7095.905 & 7095.425 & $\mathrm{FeI}(1105)$ & $4.21-5.96$ & -2.221 & 337.4 & 0.479 & 36.21 \\
\hline 7098.1 & 7097.655 & TiI & $3.30-5.30$ & -3.423 & 16.88 & 0.445 & 34.74 \\
\hline 7102.397 & 7101.932 & NiI & $4.54-6.29$ & -1.941 & 53.25 & 0.464 & 35.55 \\
\hline 7103.904 & 7103.15 & $\mathrm{FeI}(167)$ & $2.43-4.18$ & -4.488 & 18.39 & & \\
\hline 7108.084 & 7107.468 & $\mathrm{FeI}(1005)$ & $4.19-5.94$ & -1.317 & 11.32 & 0.449 & 34.87 \\
\hline 7109.397 & 7109.397 & unid & & & 8.62 & & \\
\hline 7111.541 & 7110.905 & $\operatorname{NiI}(64)$ & $1.93-3.68$ & -3.042 & 70.85 & 0.648 & 43.29 \\
\hline 7117.991 & 7117.991 & unid & & & 23.54 & & \\
\hline 7122.867 & 7122.191 & NiI(126) & $3.54-5.29$ & -0.169 & 55.08 & 0.676 & 44.40 \\
\hline 7125.038 & 7124.47 & $\operatorname{CoI}(53)$ & & & 14.34 & & \\
\hline 7125.752 & 7125.283 & $\mathrm{FeI}(1220)$ & $4.60-6.34$ & -1.465 & 32.71 & 0.468 & 35.65 \\
\hline 7126.566 & 7126.19 & $\mathrm{CaI}$ & $6.02-7.77$ & -0.647 & 60.47 & 0.375 & 31.74 \\
\hline 7419.082 & 7418.668 & $\mathrm{FeI}(1001)$ & $4.12-5.79$ & -1.17 & 107.8 & 0.414 & 32.62 \\
\hline 7420.15 & 7419.31 & $\mathrm{NiI}(287)$ & $5.50-7.17$ & -0.695 & & 0.839 & 49.84 \\
\hline 7422.671 & 7422.30 & $\mathrm{NiI}(139)$ & $3.69-5.28$ & +0.06 & 57.36 & 0.371 & 30.88 \\
\hline
\end{tabular}


Table 1. continued

\begin{tabular}{|c|c|c|c|c|c|c|c|}
\hline $\begin{array}{l}\lambda \text { obs } \\
(\AA)\end{array}$ & $\begin{array}{l}\lambda \text { lab } \\
(\AA)\end{array}$ & ID & $\begin{array}{l}\chi \\
(\mathrm{eV})\end{array}$ & $\log g f$ & $\begin{array}{l}F_{\lambda}{ }^{*} \\
(\mathrm{~m} \AA)\end{array}$ & $\begin{array}{l}\Delta \lambda \\
(\AA)\end{array}$ & $\begin{array}{r}V_{\text {Helio }} \\
\mathrm{km} \mathrm{s}^{-1}\end{array}$ \\
\hline 7430.991 & 7430.5 & $\mathrm{FeI}(204)$ & $2.58-4.24$ & -3.81 & 21.02 & 0.491 & 35.71 \\
\hline 7432.698 & 7431.97 & $\operatorname{TiI}(142)$ & $1.74-3.41$ & -2.283 & 15.53 & 0.728 & 45.27 \\
\hline 7435.814 & 7435.3 & unid & & & 47.41 & & \\
\hline 7437.521 & 7437.16 & $\operatorname{CoI}(53)$ & $1.95-3.61$ & -3.64 & 45.96 & 0.360 & 30.43 \\
\hline 7440.984 & 7440.6 & $\operatorname{TiI}(225)$ & $2.25-3.90$ & -1.19 & 38.7 & 0.383 & 31.35 \\
\hline 7446.188 & 7445.70 & FeI(1077) & $4.24-5.90$ & -0.31 & 61.72 & 0.487 & 35.53 \\
\hline 7449.784 & 7449.34 & $\operatorname{FeII}(73)$ & $3.87-5.53$ & -3.60 & 316.5 & 0.444 & 33.77 \\
\hline 7450.505 & 7450.33 & YII(?) & & & & 0.174 & 22.92 \\
\hline 7452.982 & 7452.50 & FeII $(14 \mathrm{~F})$ & & & 54.88 & & \\
\hline 7462.061 & 7461.527 & $\mathrm{FeI}(204)$ & $2.55-4.20$ & -3.48 & & 0.534 & 37.36 \\
\hline 7462.889 & 7462.38 & FeII(73) & $3.87-5.53$ & -2.98 & 540.5 & 0.509 & 36.35 \\
\hline 8327.273 & 8327.061 & $\mathrm{FeI}(60)$ & $2.20-3.69$ & -1.298 & 307.8 & 0.212 & 23.79 \\
\hline 8330.807 & 8330.587 & unid & & & 55.59 & & \\
\hline 8332.142 & 8331.926 & $\mathrm{FeI}(1153)$ & $4.39-5.88$ & -1.020 & 36.98 & 0.215 & 23.89 \\
\hline 8335.342 & 8335.150 & $\mathrm{CI}(10)$ & $7.70-9.19$ & -0.420 & 69.24 & 0.191 & 23.01 \\
\hline 8339.584 & 8339.398 & $\mathrm{Fe}(1153)$ & $4.44-5.93$ & -1.421 & 51.58 & 0.185 & 22.80 \\
\hline 8349.31 & 8349.02 & $\mathrm{FeI}(12)$ & $0.91-2.40$ & -5.605 & 106.4 & 0.290 & 26.54 \\
\hline 8364.508 & 8364.243 & TiI(33) & $0.83-2.32$ & -1.652 & 28.96 & 0.264 & 25.61 \\
\hline 8365.885 & 8365.642 & $\mathrm{FeI}(623)$ & $3.25-4.74$ & -2.040 & 110.0 & 0.243 & 24.84 \\
\hline 8675.626 & 8674.751 & $\mathrm{FeI}(339)$ & $2.82-2.42$ & -1.89 & 100.7 & 0.875 & 46.10 \\
\hline 8676.22 & 8675.38 & $\operatorname{TiI}(68)$ & $1.06-2.50$ & -1.357 & 43.2 & 0.839 & 44.88 \\
\hline 8689.396 & 8688.632 & $\mathrm{FeI}(60)$ & $2.17-3.59$ & -1.41 & 393.9 & 0.764 & 42.24 \\
\hline 8693.139 & 8692.34 & $\operatorname{TiI}(68)$ & $1.04-2.46$ & -1.92 & 27.48 & 0.798 & 43.41 \\
\hline 8727.923 & 8727.4 & {$[\mathrm{CI}]$} & & & 302.5 & 0.522 & 33.80 \\
\hline 8735.521 & 8734.70 & $\operatorname{TiI}(68)$ & $1.05-2.48$ & -2.087 & & 0.821 & 44.05 \\
\hline
\end{tabular}

were noticed in other post-AGB supergiants (García-Lario et al. 1997; Hrivnak 1997).

\section{Atmospheric parameters and chemical composition}

The UV (IUE) low resolution spectrum of HD 101584 matches well with that of an A6Ia star (HD 97534) (Fig. 9) indicating a $T_{\text {eff }}$ of $8400 \mathrm{~K}$ (Lang 1992). The presence of CII lines at $6578 \AA$ and $6582 \AA$ indicates a $T_{\text {eff }}>$ $8000 \mathrm{~K}$. For $T_{\text {eff }} \leq 8000 \mathrm{~K}$ the CII lines would be very weak or absent. The Paschen lines also indicates a low gravity (Fig. 10). The luminosity class Ia also indicates a very low gravity. From the analysis of several nitrogen lines around $7440 \AA$ and $8710 \AA$ we derived the microturbulence velocity $V_{\text {turb }}=13 \mathrm{~km} \mathrm{~s}^{-1}$. We synthesised the spectral region from $4000 \AA$ to $4700 \AA$ (Fig. 11) with low gravity ( $\log g=1.5)$ models of Kurucz (1993) with temperatures $8000 \mathrm{~K}, 8500 \mathrm{~K}$ and $9000 \mathrm{~K}$. The best fit was found for $T_{\text {eff }}=8500 \mathrm{~K}, \log g=1.5, V_{\mathrm{T}}=13 \mathrm{~km} \mathrm{~s}^{-1}$ and $[\mathrm{Fe} / \mathrm{H}]=0.0$.

The line at $5876 \AA$ was identified as a HeI line by Bakker et al. (1996a) who also state that the lines at $5047 \AA$ and $5045 \AA$ as due to HeI and NII respectively. However, we find that the 5047 and 5045 lines are in fact due to FeII. Except HeI $5876 \AA$ A, we have not found any other helium lines in the spectrum and nor have we found any NII or OII lines. In fact, Hibbert et al. (1991) indicate the presence of a CI line at $5876 \AA$. It is likely that the line at $5876 \AA$ may be due to CI instead of HeI.

If we assume that the $5876 \AA$ line is due to HeI then for a solar helium abundance and $\log g=1.5, T_{\text {eff }}=9000 \mathrm{~K}$ is found. Since we do not see any other helium lines, if $5876 \AA$ line is due to helium, it is likely that it may be formed in the stellar wind or in the chromosphere of the star. On the basis of the presence of this helium line Bakker et al. (1996a) suggested that HD 101584 is a B9II star of $T_{\text {eff }} 12000 \mathrm{~K}$. On the basis of the analysis of our spectra we have not found any evidence for such a high temperature. We have also analysed the equivalent widths of absorption lines in the spectrum of HD 101584 given by Bakker et al. (1996a). The final abundances of some of the elements are listed in Table 2. The abundances listed in Table 2 show that the star is overabundant in carbon and nitrogen. It appears that the material processed by the triple alpha $\mathrm{C}-\mathrm{N}$ and $\mathrm{O}-\mathrm{N}$ cycle has reached the surface.

\section{Discussion and conclusions}

The optical spectrum of the post-AGB star HD 101584 is rather complex. We find several emission lines and P-Cygni profiles indicating an ongoing mass-loss and the presence of a circumstellar gaseous envelope. From the analysis of the absorption lines we find the atmospheric 


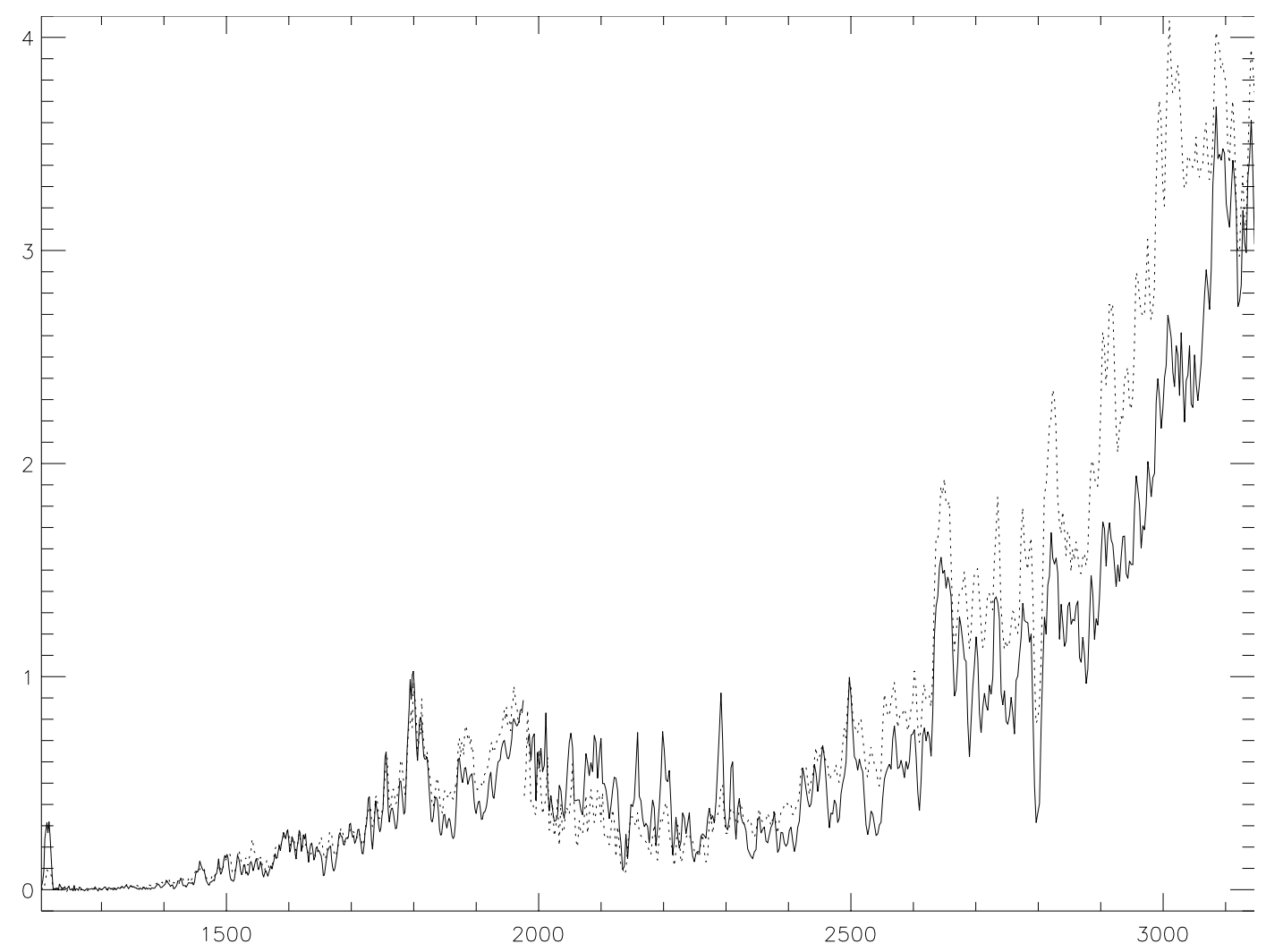

Fig. 9. IUE low resolution spectrum of HD 101584 is compared with that the A6Ia star HD 97534. The dotted line corresponds to the spectrum of HD 97534 while the solid line is HD 101584

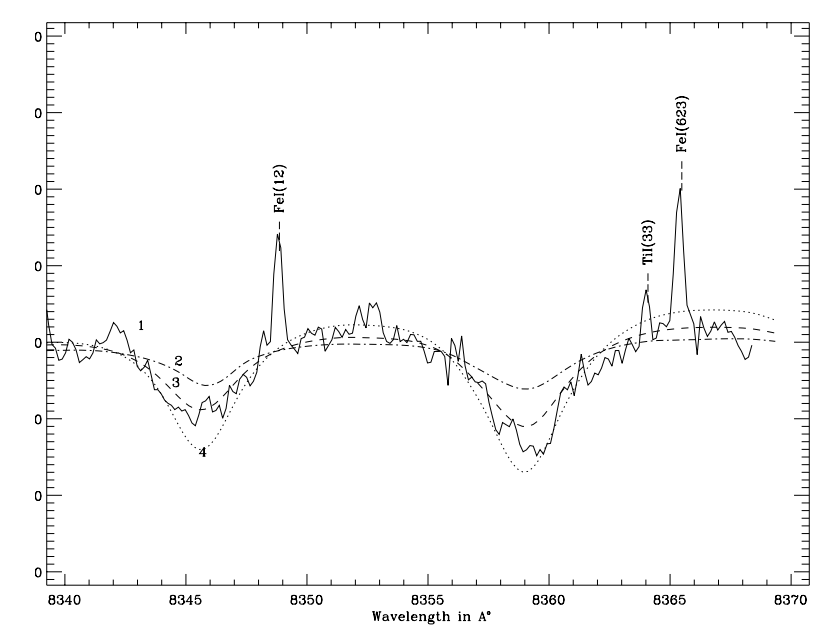

Fig. 10. Observed and synthetic spectra in the Paschen line region. 1-observed, $2-T_{\text {eff }}=8000 \mathrm{~K}, \log g=1.0,3-\left(T_{\text {eff }}=\right.$ $8000 \mathrm{~K}, \log g=2.0,4-T_{\text {eff }}=8500 \mathrm{~K}, \log g=1.5$. The peaks are FeI emission lines

parameters to be $T_{\text {eff }}=8500 \mathrm{~K}, \log g=1.5, V_{\mathrm{t}}=$ $13 \mathrm{~km} \mathrm{~s}^{-1}$ and $[\mathrm{Fe} / \mathrm{H}]=0.0$.

Carbon and Nitrogen are found to be overabundant indicating that material processed by triple alpha $\mathrm{C}-\mathrm{N}$ and $\mathrm{O}-\mathrm{N}$ cycles has reached the surface. Since our blue spectra are of relatively low resolution and because of the presence of emission and shell components it is difficult to estimate reliable abundances of s-process elements. The OI line at $6156 \AA$ is blended with a weak FeI emission line. The OI triplet at $7777 \AA$ is very strong and affected by NLTE. In any case it appears that the oxygen abundance is nearly solar. A NLTE analysis of the high resolution OI $7777 \AA$ triplet may yield a more reliable oxygen abundance.

The nitrogen abundance is based on 6 lines in the $7440 \AA$ and $8710 \AA$ region. We have not used the strong nitrogen lines. Nitrogen seems to be clearly overabundant. The carbon abundance is based on two CII lines at $6578 \AA$ and $6582 \AA$. There is a clear indication that carbon is overabundant. The abundance of $\mathrm{Mg}, \mathrm{Ti}$, and Fe are nearly solar. The Ti abundance is based on 15 lines and the Fe abundance is based on 6 lines. Many of the other atomic lines are affected by emission and shell components. In our opinion, the line at $5876 \AA$ might be due to CI (Hibbert et al. 1991) and not to HeI, as previously suggested by Bakker et al. (1996a). We have not found any other HeI, NII or OII lines. Our analysis shows that the $T_{\text {eff }}$ is $8500 \pm 500 \mathrm{~K}$.

Bakker et al. (1996b) found small amplitude light and velocity variations and suggested that HD 101584 is a binary with an orbital period of 218 days. The radial velocity variations may be due to pulsation, macroturbulence motions or shock waves in the outer layers of the stellar atmosphere. Many post-AGB supergiants show small 


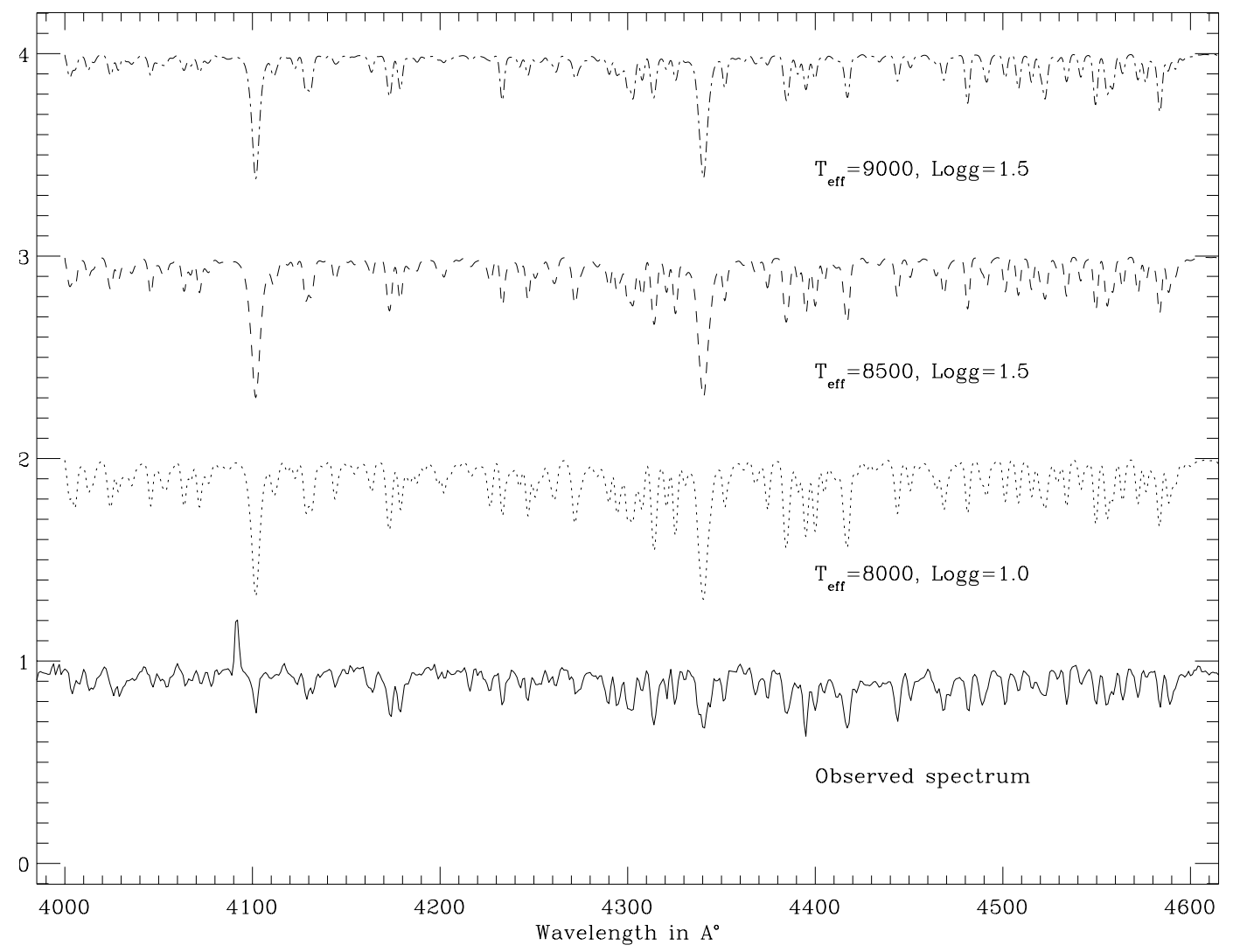

Fig. 11. Synthesis spectra for different models are compared with the observed spectrum. The observed spectrum is of $2.5 \AA$ resolution taken at VBO kavalur. Observed spectrum matches well for $T_{\text {eff }}=8500 \mathrm{~K}, \log g=1.5, V_{\text {turb }}=13 \mathrm{~km} \mathrm{~s}{ }^{-1}$ and $[\mathrm{Fe} / \mathrm{H}]=0.0$

Table 2. Chemical composition of HD 101584

\begin{tabular}{l|r|c|l}
\hline \hline \multirow{2}{*}{ Element } & \multicolumn{2}{|c|}{ [element/H] } & Lines \\
\cline { 2 - 3 } & $\begin{array}{r}T_{\text {eff }}=8500 \mathrm{~K} \\
\log g=1.5\end{array}$ & $\begin{array}{c}T_{\text {eff }}=9000 \mathrm{~K} \\
\log g=1.5\end{array}$ & \\
\hline $\mathrm{C}$ & $1.0 \pm 0.1$ & $0.3 \pm 0.1$ & C II 6578,6582 \\
$\mathrm{~N}$ & $0.5 \pm 0.1$ & $0.6 \pm 0.1$ & N I 7423, 7442, 8703, \\
& & & $8711,8718,8728$ \\
& & & \\
$\mathrm{O}$ & 0.1 & 0.18 & O I 6158 \\
& & & \\
$\mathrm{Mg}^{1}$ & $0.0 \pm 0.4$ & $0.4 \pm 0.4$ & Mg II 4481.13 \\
$\mathrm{Ti}^{1}$ & $-0.1 \pm 0.1$ & $0.5 \pm 0.1$ & Fe I 3 lines \\
$\mathrm{Fe}^{1}$ & $0.2 \pm 0.4$ & $0.3 \pm 0.4$ & Fe II 6lines \\
\hline \hline
\end{tabular}

amplitude light and velocity variations (Hirvnak 1997). These variations may not be interpreted as due to the presence of a binary companion. Long term monitoring of the radial velocities is needed in order to understand the causes for these variations.

The spectrum and the brightness of HD 101584 appears to remain the same during last two or three decades.
There is no evidence for significant variations in brightness similar to those observed in Luminous Blue Variables (LBVs). The chemical composition and all the available multiwavelength observational data collected during the last two decades by various observers indicates that HD 101584 is most likely a post-AGB star.

The presence of several P-Cygni lines with significant outflow velocities, the $\mathrm{OH}$ maser and $\mathrm{CO}$ emission profiles (Te Lintel Hekkert et al. 1992; Trams et al. 1990) and the IRAS infrared fluxes and colours (Parthasarathy \& Pottasch 1986) indicates the possibility that HD 101584 is a post-AGB star with a bipolar outflow with a dusty disk. Since HD 101584 shows a strong $\mathrm{H} \alpha$ emission line, high resolution imaging with the Hubble Space Telescope (HST) may reveal the bipolar nebula and the presence of a dusty disk similar to that observed in other post-AGB stars like IRAS 17150-3224 (Kwok et al. 1998) or IRAS 17441-2411 ( $\mathrm{Su}$ et al. 1998).

\section{References}

Bakker E.J., 1994, A\&AS 103, 189

Bakker E.J., Lamers H.J.G.L.M., Waters L.B.F.M., Waelkens C., Trams N.R., Van Winckel H., 1996a, A\&A 307, 869 
Bakker E.J., Lamers H.J.G.L.M., Waters L.B.F.M., Waelkens C., 1996b, A\&A 310, 861

Friedjung M., Muratorio G., 1987, A\&A 188, 100

García-Lario P., Parthasarathy M., de Martino D., Monier R., Manchado A., de Córdoba S.F., Pottasch S.R., 1997, A\&A 326,1103

García-Lario P., Manchado A., Parthasarathy M., Pottasch S.R., 1994, A\&A 285, 179

Hibbert A., Biémont E., Godefroid M., Vaeck N., 1991, A\&AS 88,505

Hrivnak B., 1997, in Planetary Nebulae, Habing H.J. and Lamers H.J.G.M. (eds.), IAU Symp. No. 180, 303

Hoffleit D., Saladya M., Wlasuk P., 1983, Supplement to the Bright star Catalogue, Yale University Observatory, U.S.A.

Hubeny I., Stefl S., Harmanec P., 1985, Bull. Astron. Inst. Czechosl. 36, 214

Humphreys R.M., 1976, ApJ 206, 122

Humphreys R.M., Ney E.P., 1974, ApJ 190, 339

Kurucz R.L., 1994, Solar Abundance Model Atmospheres, Kurucz CD-ROM No. 19, Smithsonian Astrophysical Observatory

Kwok S., Su K.Y.L., Hrivnak B.J., 1998, ApJ 501, L117

Lang K.R., 1992, Astrophysical data: Planets and stars.
Springer Verlag

Morrison N.D., Zimba J.R., 1989, BAAS 21, 1022

Osterbrock D.E., 1989, Astrophysics of Gaseous Nebulae and Active Galactic Nuclei. Oxford University Press, p. 117

Parthasarathy M., García-Lario P., Pottasch S.R., 1992, A\&A 264, 159

Parthasarathy M., Pottasch S.R., 1986, A\&A 154, L16

Reddy B.E., Parthasarathy M., Gonzalez G., Bakker E.J., 1997, A\&A 328, 331

Rosenzweig P., Reinoso E.G., Naranjo O., 1997, JRASC 91, 255

Su K.Y.L., Volk K., Kwok S., Hrivnak B.J., 1998, ApJ 508, 744

Te Lintel Hekkert P., Chapman J.M., Zijlstra A.A., 1992, ApJ 390, L23

Trams N.R., Van der Veen W.E.C.J., Waelkens C., Waters L.B.F.M., 1990, A\&A 233, 153

Viotti R., 1969, Astrophys. Space Sci. 5, 323

Wiese W.L., Smith M.W., Glennon B.M., Atomic Transition Probabilities, Vol. 1, NSRDS-NBS(U.S.) 4, 1966

Wiese W.L., Martin G.A., Wavelength and Transition Probabilities for Atoms and Atomic Ions, NSRDSNBS(U.S.) 68, 1980 